\title{
BMJ Open Biomarkers of exposure and early effect in three contaminated sites of southern Italy: protocols for etiological epidemiological studies
}

\author{
Francesca Gorini (D) , ${ }^{1}$ Elisa Bustaffa, ${ }^{1}$ Davide Bolignano, ${ }^{2}$ Liliana Cori, ${ }^{1}$ \\ Francesco Faita, ${ }^{1}$ Amalia Gastaldelli, ${ }^{1}$ Monica Interdonato, ${ }^{3}$ Fabizio Minichilli, ${ }^{1}$ \\ Giancarlo Quattrone, ${ }^{4}$ Francesco Squadrito, ${ }^{3}$ Giovanni Tripepi, ${ }^{2}$ Cristina Vassalle, ${ }^{5}$ \\ Fabrizio Bianchi ${ }^{1}$
}

To cite: Gorini F, Bustaffa E, Bolignano D, et al. Biomarkers of exposure and early effect in three contaminated sites of southern Italy: protocols for etiological epidemiological studies. BMJ Open 2020;10:e036160. doi:10.1136/ bmjopen-2019-036160

- Prepublication history for this paper is available online. To view these files, please visit the journal online (http://dx.doi. org/10.1136/bmjopen-2019036160).

Received 03 December 2019 Revised 19 March 2020 Accepted 03 April 2020

Check for updates

(C) Author(s) (or their employer(s)) 2020. Re-use permitted under CC BY-NC. No commercial re-use. See rights and permissions. Published by BMJ.

For numbered affiliations see end of article.

Correspondence to

Francesca Gorini;

fgorini@ifc.cnr.it

\section{ABSTRACT}

Introduction Environmental pollution has been progressively becoming one of the main risk factors to human diseases. In particular, populations living in high-contaminated sites are particularly exposed to environmental toxicants, with consequent increased risks to human health. In Italy, there are currently ongoing three epidemiological etiological studies aimed at evaluating the association between exposure to inorganic and organic chemicals and presence of biological markers of early effects in population living in three National Priority Contaminated Sites (NPCSs). Specifically, the correlations concern preclinical indicators of liver disease in Priolo NPCS, thyroid diseases in Milazzo-Valle del Mela NPCS and cardiovascular risk and kidney damage in Crotone NPCS.

Methods and analysis Overall, approximately 1300 subjects of both sexes will be enrolled in the three NPCSs according to specific inclusion criteria. For each subject, serum and urine specimens are collected, on which the determination of biological markers of exposure and early effects for the selected outcomes are performed. Individual information on environmental and occupational exposure, medical history, diet and life habits is obtained through questionnaires provided by web platform. In Milazzo-Valle del Mela and Crotone NPCSs, not invasive instrumental and imaging examinations are performed in order to evaluate further risk factors of thyroid carcinoma and cardiovascular disease, respectively.

Ethics and dissemination The protocol studies have been approved by the Ethics Committees responsible for the three involved NPCSs: the Ethics Committee 'Catania 2' for the NPCS of Priolo (21 July 2017, n. 500/2017/ CECT2), the Ethics Committee of the University Hospitals of Messina for the NPCS of Milazzo-Valle del Mela (19 February 2018, n.2/2018); the Ethics Committee of the Region of Calabria for the NPCS of Crotone (20 July 2017, n. 174). Results will be disseminated among policy-makers, citizens, stakeholders and scientific community through the organisation of conferences and events, and the publication on international peer/ reviewed journals.

\section{Strengths and limitations of this study}

- In the framework of the CISAS project epidemiological studies based on the evaluation of biological markers of exposure and early effects in populations living in high-contaminated sites of southern Italy are proposed.

- The levels of exposure and the presence of risk factors and early-effect indicators of selected outcomes are evaluated by using biohumoural tests, instrumental and imaging examinations and information by individual questionnaire.

- The sample size in the three studies is representative of the subpopulation with the distribution by sex, age class and municipality, also considering inclusion/exclusion criteria as established by the study protocols, and it has been defined to evaluate statistically significant differences in biological markers between the study and the reference areas.

- The cross-sectional study design is the weakest of the etiological studies, but it is sufficiently informative to investigate the relationship between environmental exposure and early indicators of disease.

- The biological markers of health risk, although predictive of important health outcomes, are affected by limits of precision and accuracy proper of biomarkers commonly used in clinical practices.

\section{INTRODUCTION}

In the last decades, research on environment and health has progressively gained importance for contemporary society, given the faster and faster environmental changes and the consequent threats to human health. In 2012, it has been estimated that 12.6 million deaths globally, representing $23 \%$ of all deaths and $26 \%$ of deaths among children under 5 years, were attributable to environmental exposures. ${ }^{1}$

The Sixth Ministerial Conference on Environment and Health of 53 Countries 
of the WHO European Region, included for the first time contaminated sites as leading environmental risks and consequently among the priority actions for public health. ${ }^{2}$ In the all EU Member States, the total number of potentially contaminated sites is estimated in 2.8 million, of which 65500 have been already remediated or are under aftercare measures, representing an increment of more than 8500 new remediated sites in the last 5 years. ${ }^{3}$ Nonetheless, since 2011, a reduction in the number of sites under remediation has been reported by Belgium, Estonia, Italy, Latvia, Norway and Slovakia. ${ }^{3}$ The contamination of these areas particularly concerns soils and water for which industrial and commercial activities as well as waste disposal and treatment represent the main sources of pollution. ${ }^{4}$ As regards air pollution, over the period 2008-2012, considering a total of 14325 single facilities in the EU-27, plus Norway and Switzerland, the cost of damage to health and environment produced by their emissions ranged between 329 and 1065 billion euros. ${ }^{5}$ It is important to note that $50 \%$ of the total damage cost occurs as a result of emissions from 147 plants, and countries such as Germany, Poland, the UK, France and Italy, having a high number of large facilities, contribute the most to total estimated damage costs. ${ }^{5}$ Air pollutants (carbon dioxide, nitrogen oxides, sulphur dioxide and particulate matter), heavy metals (eg, cadmium (Cd), lead $(\mathrm{Pb})$, arsenic (As) and mercury) and organic compounds (benzene, dioxins and furans, polycyclic aromatic hydrocarbons) represent the main environmental determinants used to quantify the impacts and associated damage costs. ${ }^{5}$

In Italy, there are currently 41 National Priority Contaminated Sites (NPCSs), with a total surface area of $1712 \mathrm{~km}^{2}$ and representing $0.57 \%$ of the national surface area. NPCSs are identified as highly contaminated areas by the Ministry of Environment, in agreement with the regions concerned. ${ }^{6}$

The Italian Studio Epidemiologico Nazionale dei Territori e degli Insediamenti Esposti a Rischio da Inquinamento (SENTIERI) project, initiated in 2006, provided an ecological epidemiological observation of the populations residing in the aggregation of municipalities included in each NPCS. ${ }^{7}$ The current update of SENTIERI covers 45 sites, which include 319 municipalities, with a total population of approximately 5900000 inhabitants $(9.8 \%$ of national population). ${ }^{4}$ An overall excess of 5267 and 6725 deaths due to all natural causes was detected, respectively, in the male and female population, while the excess due to oncological causes was equal to 3375 men and 1910 women. ${ }^{4}$ The study is based on the a priori identification of the health effects expected to be associated to the main source of pollution (ie, industrial plants, harbours, landfills and incinerators), and provided the first solid basis for a global evaluation of the impact of industrial areas on the health of exposed communities. Nonetheless, its ecological design and the consequent use of aggregated data both for exposure and health indicators does not allow to infer any causal relationship between exposures and health outcomes. ${ }^{8}$
With the aim of studying the relationships in depth between environment and health in selected NPCSs, the Italian Ministry of Education, Universities and Research has funded the ongoing 'International Centre of Advanced Study in Environment, Ecosystem and Human Health' (CISAS) project, which involves four institutes of the National Research Council, in collaboration with scientific and regulatory bodies (http://www.cisas.cnr. it/). CISAS is a multidisciplinary study aimed at understanding environmental pollution phenomena and their impact to ecosystem and human health in highly contaminated sites, organised in six working packages (WPs). The research activity, to be developed by 2021, is focused on the three NPCSs of Priolo and Milazzo-Valle del Mela (Sicily, Italy), and Crotone (Calabria, Italy), characterised by severe environmental contamination and relating health impacts.

The environmental and health studies currently in progress have the objective to assess the levels of contamination of air, water and soil, the routes of chemicals in the environmental matrices, the exposure profiles of local communities through the main route of exposures, including the consumption of food of local production, in addition to investigate the effects of toxicants on pregnancy outcomes in a newborn cohort study. ${ }^{9}$

The Institute of Clinical Physiology of the National Research Council (IFC-CNR) is coordinating a WP developing epidemiological studies with etiological design aimed at evaluating the relationship of exposure to specific pollutants with selected early markers exposure or health risk. Such indicators were identified on the basis of the existing scientific evidence of association with environmental pollutants and the relevance for research and innovation. These studies, in order to assess environmental exposure, consider residence in exposed or unexposed area and the measurement of individual exposure markers. Hereinafter, we describe rationale, objectives and results expected for each planned study.

\section{METHODS AND ANALYSIS}

Experimental context

NPCS of Priolo

Priolo NPCS, other than the Gela and Milazzo NPCSs, is defined by the Sicilian Region as a 'high-crisis environmental area', being characterised by the presence of major industrial settlements, mainly petrochemical plants, which in the last decades have caused a progressive contaminations of environmental matrices. In this area, high concentrations of persistent and bioaccumulative toxic compounds, such as heavy metals, polychlorinated biphenyls (PCBs), dioxins, polycyclic aromatic hydrocarbons and volatile organic compounds, have been detected. ${ }^{10}$ Meanwhile, there has been increased incidence of several types of tumours, in particular mesothelioma, liver and pancreatic neoplasms, in the population of both sexes living in the NPCS, if compared with the rate recorded in Cancer Registries of Central-Southern 
Italy. ${ }^{11}$ Hepatocellular carcinoma accounts for $>90 \%$ of primary liver cancer cases ${ }^{12}$ and it is currently the third leading cause of cancer deaths worldwide (https://emedicine.medscape.com/article/197319-overview). In addition to alcoholism, hepatitis $\mathrm{B}$ virus, hepatitis $\mathrm{C}$ virus and illness conditions including non-alcoholic fatty acid liver disease (NAFLD), obesity, type 2 diabetes, cirrhosis, ${ }^{13}$ the exposure to endocrine disrupting chemicals able to induce metabolic alterations, particularly on lipids and consequent lipotoxicity and oxidative stress ${ }^{14}$ represents a further risk factor for hepatocellular Carcinoma (HCC). ${ }^{1315}$

\section{NPCS of Milazzo-Valle del Mela}

The high-level contamination of the area is primarily attributable to the presence of plants for the electricity production, a refinery, a steel plant and fuel oil power plant. At global level, the last three decades have been characterised by a significant increase of thyroid carcinoma, the most frequent endocrine malignancy, and, among the acknowledged risk factors including age, sex, radiation, a familiar history of thyroid cancer, ${ }^{16}$ much evidence supports the likely etiological role of organic compounds (ie, PCBs, polybrominated diphenyl ethers, phthalates and bisphenols) and heavy metals (ie, Cd and $\mathrm{Pb}$ ) that may act as thyroid disruptors through multiple mechanisms such as interference with thyroid hormone (TH) synthesis, TH metabolism and excretion, and TH action, ${ }^{17}$ which, however, have not been completely elucidated. ${ }^{18} 19$ In the early 2000s, a higher incidence and hospitalisation for thyroid cancer was observed in the population living in the NPCS of Milazzo when compared with the regional rate. ${ }^{11}$ Recently, a biomonitoring study reported that adolescents living in the contaminated area exhibit a significantly more elevated concentration of urinary $\mathrm{Cd}$ than peer controls. ${ }^{20}$ Exposure to $\mathrm{Pb}$ and $\mathrm{Cd}$, in particular, was reported to be related to thyroid dysfunction in adult women, ${ }^{21}$ while Cd levels were higher in the blood and thyroid tissue of patients with thyroid cancer. ${ }^{22} 23$

\section{NPCS of Crotone}

The past presence of industrial activities, namely for production of zinc, phosphates and nitrogen fertilisers, resulted in a widespread contamination by heavy metals and hydrocarbons in this site. ${ }^{7}$ In this area, an excess of mortality for kidney cancer in both sexes, and for urinary tract infections and kidney failure in women were observed. In addition, increased hospitalisation rate was found in men and women for ischaemic heart disease, nephritis and nephrotic syndrome, while a higher rate of renal tumours emerged among males. ${ }^{24}$ There are increasing suggestions that exposure to As and other toxic metals may be an independent risk factor for cardiovascular disease (CVD) ${ }^{25}{ }^{26} \mathrm{~A}$ recent systematic review and meta-analysis reported that exposure to $\mathrm{As}, \mathrm{Cd}, \mathrm{Pb}$ and copper is significantly and positively associated with CVD, coronary heart disease and stroke, and for As, Cd and $\mathrm{Pb}$ a linear dose-response relationship was observed. The mechanisms involved comprise induced oxidative stress, inflammation and endothelial cell damage, which can lead to atherosclerosis. ${ }^{27}$ Furthermore, the kidney is a target organ of heavy metal toxicity for its capability to reabsorb and concentrate divalent ions and metals. ${ }^{28}$ $\mathrm{Pb}$ and $\mathrm{Cd}$ are established nephrotoxins at high exposure levels ${ }^{29}$ and even at environmental exposure may contribute to the risk of chronic kidney disease, a progressive and irreversible deterioration in the renal function, especially in adults with hypertension or diabetes. ${ }^{30} 31$

\section{Study design}

The three epidemiological studies have a cross-sectional design and are based on the measurement of exposure and early-effect biological markers. For each subject recruited, the studies provide for the following:

- The administration of a questionnaire aimed at gathering anthropometric data, information on residential history, socioeconomic and health conditions, environmental and occupational exposure, lifestyle, diet, risk perception.

- The collection of biological samples (serum and urine).

- The measurement of systolic and diastolic blood pressure and heart rate, and neck ultrasound in MilazzoValle del Mela NPCS.

- Carotid ultrasound, brachial artery flow-mediated dilation, arterial tonometry, ECG in Crotone NPCS.

\section{Definition of study and reference areas}

The NPCS of Priolo (defined by the Decree 426/1998 of the Italian Ministry of the Environment) comprises the municipalities of Augusta, Melilli, Priolo Gargallo and Syracuse (184.308 total inhabitants on 1 January 2016, http://www.demo.istat.it/index.html). The latter has been excluded from the study area as its territorial and demographic characteristics are very different from the other municipalities included in the NPCS, namely it has a much larger population (122291 total inhabitants) and only a small part of the resident population is resident close to the boundaries of the perimeter of the NPCS. Furthermore, differently from the other municipalities that are homogeneous in both demographic and environmental characteristics, inhabitants of Syracuse are exposed to multiple sources of pollution with a significant component dependent on heating and vehicular traffic. Two other municipalities, Noto and Avola (55621 total inhabitants on 1 January 2016, http:/ /www.demo.istat.it/ index.html) were included in the reference area because of the similar demographic and territorial characteristics to those of the study area, that is, located along the coast and not interested by environmental issues of industrial origin (figure 1).

The NPCS of Milazzo-Valle del Mela (defined by the Decree 266/2005 of the Italian Ministry of the Environment) includes the municipalities of Milazzo, San Filippo del Mela, Pace del Mela (about 45000 total inhabitants on 


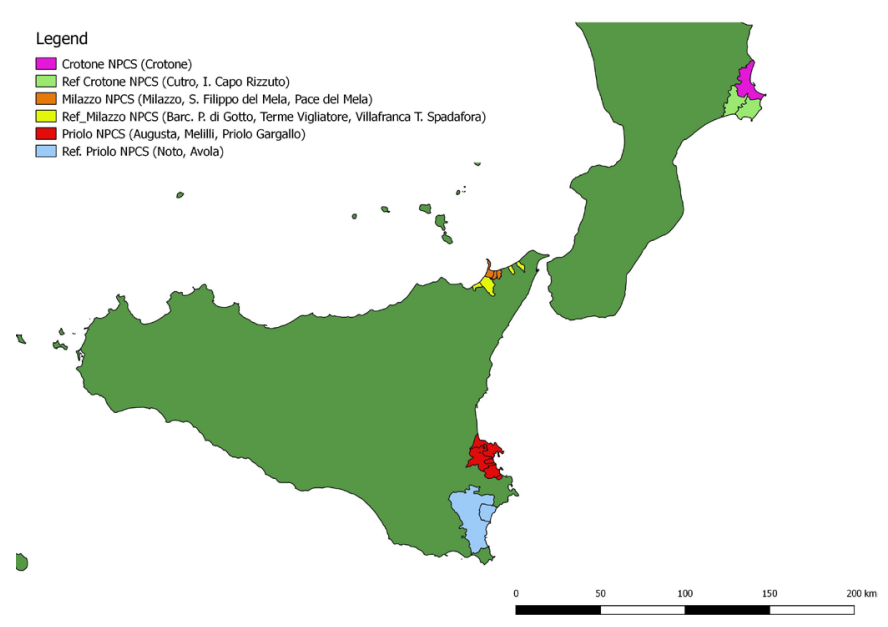

Figure 1 Map of National Priority Contaminated Sites (NPCS) and reference areas in CISAS project. Created through QGIS 2.18 Development Team. QGIS Geographic Information System. Open Source Geospatial Foundation. URL: https://www.qgis.org/it/site/forusers/ visualchangelog218/index.html

1 January 2016, http://www.demo.istat.it/index.html). Other four municipalities (Barcellona Pozzo di Gotto, Terme Vigliatore, Villafranca Tirrena and Spadafora) were considered as reference area (62583 total inhabitants on 1 January 2016, http:/ /www.demo.istat.it/index. html) (figure 1).

As regards the NPCS of Crotone, defined by the Decree $468 / 2001$ of the Italian Ministry of the Environment (total inhabitants 62178 on 1 January 2016, http://www. demo.istat.it/index.html), two municipalities, Cutro and Isola di Capo Rizzuto (total inhabitants 28.184 on 1 January 2016, http://www.demo.istat.it/index.html), were selected as reference area (figure 1).

\section{Inclusion criteria for recruitment}

For the three epidemiological studies, inclusion criteria have been established as follows:

\section{For the NPCS of Priolo:}

- Men and women aged 40-70 years (the age class most at risk for the development of liver disease). ${ }^{32}$

- Residence in the study area for at least 10 years before the enrollment: since liver disease is a long-term disease, this time of residence is considered sufficient for the evaluation of early-effect biological markers. ${ }^{33}$

- Absence of known liver diseases.

- Signed informed consent to participate in the study.

Subjects with an acknowledged diagnosis of liver disease, positivity for hepatitis B and $\mathrm{C}$ virus infections, with abuse of alcohol or consumption of other drugs are excluded from the study.

For the NPCS of Milazzo-Valle del Mela:

- Men and women aged 18-44 years (thus excluding women in menopausal status, which could lead to a misclassification of early-effect biological markers).

- Residence in the study area for at least 10 years before the enrollment. ${ }^{2034}$

- Absence of thyroid diseases.
- Signed informed consent to participate in the study.

Individuals with an acknowledged diagnosis of thyroid disease or subjected to pharmacological therapy for thyroid disease are excluded from the study.

For the NPCS of Crotone:

- Men and women aged 40-70 years (only subjects in cardiovascular primary prevention were selected due to the high sensibility and vulnerability of this population). ${ }^{35}$

- Residence in the study area for at least 5 years before the enrollment, as this period of time is optimal to link the measured CV biomarkers to the CV outcomes in terms of both mortality and morbidity. ${ }^{36} 37$

- Subjects in primary prevention for cardiovascular risk.

- Signed informed consent to participate in the study.

The exclusion criteria include the presence of systemic diseases, past cardiovascular events and/or history of nephropathy.

\section{Questionnaires}

In addition to some common sections (ie, personal data, sports, smoking and alcohol consumption, medical history and clinical conditions, environmental and socioeconomic conditions, occupational activity, exposure to physical and chemical agents, diet, reproductive history, hazard and risk perception, access to information on environment and health), the questionnaire administered to the participants includes other sections aimed at providing insights about potential sources of exposure, and previous and current diseases of subjects and their own families specific for each NPCS:

- Priolo NPCS: physical and/or sporting activity practiced and at what level, vaccinations, dental fillings, tattoos and /or piercings, family medical history, previous and current CVDs, previous and current nonCVDs, domestic heating/cooling modality, owning of televisions/tablets/computers/mobile phones.

- Milazzo-Valle del Mela NPCS: symptomatology associated to thyroid diseases, use of supplements, history of adverse reproductive outcomes and information on menstrual cycle for women, information on sterility/ infertility for both sexes.

- Crotone NPCS: sleep disorders, adverse life events, family medical history, primary factors of cardiovascular risk, previous and current CVDs, previous and current non-CVDs.

The questionnaires have been tested for comprehension and readability by three researchers independently. In order to avoid both paper administration and human errors, an online platform has been created for entering answers to the questionnaires. The questionnaires are administered by one or more operators who reported the answers on the platform. To adequately train each operator, a guide for the correct administration of the questionnaires has been produced and delivered to the contact person of each area. The questionnaires may be requested to these authors. 


\section{Biohumoural tests}

In the three NPCSs and corresponding reference areas, a sample of venous blood and a sample of urine is collected for all participants (table 1). Blood tubes are stored at $4^{\circ} \mathrm{C}$ and centrifuged within 24 hours for analyses performed locally, whereas aliquots of plasma and urine are stored at $-20^{\circ} \mathrm{C}$ until their transfer to the biobank of IFC-CNR for supplementary tests.

\section{NPCS 'Priolo'}

In this study, the results deriving from blood clinical tests are used with the anthropometric data to determine the Fatty Liver Index (FLI), the Fibrotic Liver Index (FIB-4) and the NAFLD Fibrosis Score (NFS), in order to evaluate the presence of hepatic steatosis and fibrosis, respectively. ${ }^{38-40}$ According to the European Association for the Study of the Liver, European Association for the Study of Diabetes and European Association for the Study of Obesity, FLI, FIB-4 and NFS are non-invasive markers, which can be used with liver enzymes to identify subjects at risk of liver disease. ${ }^{41}$

- The FLI is calculated by the following formula: ${ }^{39}$ $\left(\mathrm{e}^{0.953 * \operatorname{loge} \text { (triglycerides) }+0.139 * \mathrm{BMI}+0.718 * \text { loge }(\mathrm{ggt})+0.053 * \text { waist }}\right.$ circumference -15.745$) /\left(1+\mathrm{e}^{0.953 * \text { loge (triglycerides) }+0.139 * \mathrm{BMI}}\right.$ $+0.718 * \operatorname{loge}(\mathrm{ggt})+0.053 *$ waist circumference -15.745$) * 100$

with the cut-off FLI $<30$ for the presence of NAFLD.

- The FIB-4 computation is based on the following formula: ${ }^{42}$

age (years) x AST $(\mathrm{U} / \mathrm{L}) /\left(\right.$ platelets $\left(10^{9} / \mathrm{L}\right) \times$ (ALT $(\mathrm{U} / \mathrm{L})) 1 / 2$ ) with the cut-off FIB-4 $>1.30$ for the presence of fibrosis.

- The $\mathrm{NFS}^{43}$ is calculated by the following formula (http://gihep.com/calculators/hepatology/ nafld-fibrosis-score/):

$1.675+0.037 \times$ age $($ years $)+0.094 \times$ BMI $\left(\mathrm{kg} / \mathrm{m}^{2}\right)+1.13$ $\times \mathrm{IFG} /$ diabetes $($ yes $=1$, no $=0)+0.99 \times \mathrm{AST} /$ ALT ratio $0.013 \times$ platelet $\left(\times 10^{9} / \mathrm{L}\right)-0.66 \times$ albumin $(\mathrm{g} / \mathrm{dL})$ with the cut-off NFS >-1.455 for the presence of fibrosis.

Furthermore, serological lipidomics markers and urinary phthalates and bisphenols are evaluated by quadrupole time-of-flight liquid chromatography/mass spectrometry and gas chromatography/mass spectrometry, while serum inflammatory markers are measured by Luminex technology (table 1).

\section{NPCS 'Milazzo Valle del Mela'}

In addition to the determination of serum THs, namely thyroxine (T4), triiodothyronine (T3) and thyroid stimulating hormone, also the evaluation of the gene expression of nuclear receptors of TH (THR-alpha and THR-beta) and retinoic acid (RAR-alpha and RARgamma), and of 9-cis-retinoic acid receptor (RXR-alpha) levels in whole blood are performed as previously done (table 1). ${ }^{44}$ The analysis of gene expression crossed with the related hormone levels allows to assess the possible association between exposure and clinical or subclinical hypothyroidism or other subclinical conditions of alterations of THs as well. Furthermore, urinary $\mathrm{Cd}$ and $\mathrm{Pb}$, considered as markers of individual environmental exposure, are determined with Inductively Coupled Plasma Mass Spectrometry (ICP-MS) by an accredited laboratory.

\section{NPCS 'Crotone'}

Markers of inflammation, oxidative stress, cardiac biomarkers, markers of blood vulnerability to thrombosis and bone health are measured in plasma specimens of each participant (table 1). The potential association between exposure to heavy metals and CVD has several clinical implications, mainly due to an increase in oxidative stress and inflammatory status. ${ }^{45} 46$ In addition, chronic exposure to Cd can lead to increased arterial stiffness and altered endothelial function. ${ }^{47} 48$ It is interesting to note that different heavy metals are pollutants able of critically influencing bone mass ${ }^{49}$ that is associated, according to recent studies, to a higher risk of CVD. ${ }^{5051}$

The impact of environmental exposure to heavy metals on renal function is assessed through the determination of biomarkers of tubular damage in urine samples, indexed by urinary creatinine excretion levels. Moreover, kidney damage is further estimated by the glomerular filtration rate (GFR) using serum creatinine-based formulas (Cockcroft-Gault and Modification of Diet in Renal Disease) ${ }^{52}$ and serum cystatin-based formula ${ }^{53}$ for predicting GFR.

These biochemical tests are performed using standard available automated methods, or manual colorimetric and ELISA commercially available kits, according to the manufacturer's instructions.

Finally, individual exposure to $\mathrm{Cd}$ and $\mathrm{Pb}$ is determined both in blood and urine specimens with ICP-MS by an accredited laboratory.

\section{Instrumental and imaging examinations \\ NPCS 'Milazzo Valle del Mela'}

In addition to biohumoural tests, a neck ultrasonography is performed in all subjects by a commercially available system focusing on the examination of the following parameters: general characteristics of the thyroid parenchyma (glandular volume, echogenicity, echo structure, vascularisation and presence of parenchymal calcifications), morphostructural evaluation of possible nodular lesions (location, size, echogenicity, echo structure, calcifications, content and vascular pattern) and possible presence of lymphadenopathy. In the case of indications emerging from the ultrasound scan for the opportunity to proceed with fine-needle aspiration biopsy (ie, the presence of thyroid nodules with diameter $>10 \mathrm{~mm}$ or nodules $<10 \mathrm{~mm}$ in diameter that require further evaluation because of clinical symptoms or associated lymphadenopathy) ${ }^{54}$ this information is transmitted to the subject and to the attending physician.

\section{NPCS 'Crotone'}

Brachial artery flow mediated dilation is used to assess endothelial function by processing of ultrasound images of the brachial artery following International consensus guidelines. ${ }^{55}$ In particular, maximal percentage increase 
Table 1 Summary of clinical chemistry tests on blood and urine specimens by study area

\begin{tabular}{ll} 
Area & Test \\
\hline Priolo NPCS & Blood \\
& Hemochrome, FBG, triglycerides, total and HDL cholesterol, SGOT, SGPT, GGT, \\
& HbA1c, bilirubin, ferritin, transferrin, albumin \\
& Lipidomic profile (including free fatty acid profile), leptin, insulin, inflammatory \\
& biomarkers (MCP-1, TNF $\alpha)$ \\
& Total 10 \\
& Urine \\
& Measurement of phthalates and bisphenols \\
& Total
\end{tabular}

\section{Milazzo Valle del Mela NPCS Blood}

Hemochrome, ESR, fibrinogen, CRP, glycaemia, creatinine, triglycerides, total, 12

$\mathrm{HDL}$ and LDL cholesterol

TSH, FT3, FT4, thyroglobulin, antithyroglobulin Ab 6

Calcitonin 6

$\mathrm{Cd}, \mathrm{Pb} \quad 6$

THR-alpha, THR-beta, RAR-alpha, RAR-gamma, RXR-alpha 4

Total 34

Urine

Early morning urine: lodine, creatinine $\quad 10$

24 hours urine: $\mathrm{Cd}, \mathrm{Pb} \quad 10$

Total 20

\begin{tabular}{llc} 
Crotone NPCS & Blood & \\
Cardiovascular system & Hemochrome, triglycerides, total, HDL and LDL cholesterol, urea, creatinine, ALT, & 15 \\
& AST, GGT, CRP, glycaemia, fibrinogen, TSH & 1 \\
& Inflammatory markers (IL-6) & 2 \\
& Oxidative stress markers (hydroperoxides, total antioxidant capacity) & 6 \\
& Markers of blood vulnerability to thrombosis (circulating microparticles, tPA and & 3 \\
& CD40 ligand) & 3 \\
& Fibrosis (galectin-3) and cardiac damage markers (BNP) & 6 \\
& Bone health markers (vitamin D, osteocalcin) & 36 \\
& Cd, Pb & 10 \\
& Total & 10 \\
Crotone NPCS & Blood & 10 \\
kidney & Cystatin C & 10 \\
& Urine & 10 \\
& Early morning urine: markers of tubular damage (NAG, $32-M G$, KIM-1, NGAL, L- & 10 \\
& FABP) & 10 \\
& 24 hours urine: albumin, protein & 30 \\
\hline
\end{tabular}

Continued 
ALT, alanine aminotransferase; AST, aspartate aminotransferase; BNP, N-terminal pro B-type natriuretic peptide; Cd, cadmium; CRP, C reactive protein; ESR, erythrocyte sedimentation rate; FBG, fasting blood glucose; FT3, free triiodothyronine; FT4, thyroxine; GGT, gamma glutamyl transferase; HDL, high density lipoprotein; IL-6, interleukine 6; KIM-1, Kidney injury molecule-1; LDL, low density lipoprotein; L-FABP, liver-type fatty acid binding protein; MCP-1, monocyte chemotactic protein-1; $\beta 2-\mathrm{MG}, \beta 2$-microglobulin; NAG, N-acetyl- $\beta$-D-glucosaminidase; NGAL, neutrophil gelatinase-associated lipocalin; NPCS, National Priority Contaminated Sites; Pb, lead; RAR, retinoid acid receptor; RXR, retinoid X receptor; SGOT, alanine aminotransferase; SGPT, aspartate aminotransferase; THR, thyroid hormone receptor; TNF $\alpha$, tumor necrosis factor $\alpha$; tPA, tissue plasminogen activator; TSH, thyroid stimulating hormone.

of the brachial artery diameter following reactive hyperaemia is measured using an automatic edge detection system (FMD Studio, Quipu, Pisa Italy). Furthermore, carotid-femoral pulse wave velocity (PWV) is assessed as a surrogate marker of regional arterial stiffness. For this purpose, an applanation tonometer is employed to record pressure waveforms at the carotid and femoral artery levels, thus obtaining the PWV value. The carotid pressure curve is also rescaled in order to estimate central blood pressure and augmentation index. Ultrasound scans of the common carotid artery are acquired and processed in order to assess anatomical and functional parameters. In particular, intimal-media thickness, plaque presence and carotid stiffness is estimated using an automatic software (Carotid Studio, Quipu, Pisa, Italy). ${ }^{56}$ Finally, heart rate variability is performed automatically both in time and frequency domain by processing 12-lead ECG traces.

\section{Statistics}

Distribution of people residing in each study and reference has allowed to extract a sample that reflects distribution by sex, age class and municipality of residence of inhabitants in NPCSs and corresponding reference areas. Thus, from the list of patients of Local Health Companies of Siracusa, Messina and Crotone, the sample, sized in proportion to the population of every municipality included in the study, was randomly selected with stratification by sex and age class. To calculate the statistical power of each study, the following indicators were considered:

- Priolo NPCS NFS and FLI: in order to obtain a difference of -1 between the value of NFS 2.4 obtained in a general population $^{38}$ and the cut-off of NFS $=-1.45^{43}$ to distinguish the subjects with fibrosis from those without fibrosis; and a difference of -15 between the reference value of FLI $=35$ obtained in a general population $^{38}$ and the value of NFS=50 for the subjects with NAFLD, ${ }^{43}$ a sample of 500 subjects was defined, 250 in the NPCS and 250 in the reference area, with a statistical power $(1-\beta)$ of $90 \%$ and a significance level $(\alpha)$ of 0.05 using a two-tailed t-test.

- Milazzo-Valle del Mela NPCS: thyroid nodules with $>10 \mathrm{~mm}$ diameter. Palpable thyroid nodules $>10 \mathrm{~mm}$ diameter are detected in approximately $5 \%$ of the population: ${ }^{54}$ most of them are benign but $5 \%$ develops to malignant nodules. ${ }^{57}$ With a sample of 480 subjects, 240 for each area, $\alpha=0.05,1-\beta=0.8$ through bilateral test, and a difference of risk between the two areas corresponding to 0.07 , were obtained. A statistical power of $80 \%$, and not of $90 \%$ as for the other two studies, was considered acceptable given the available funds, which limit the recruitment of a higher number of cases than estimated using a power of $80 \%$.

- Crotone NPCS: arterial stiffness. This is the indicator that, among those of imaging, has the characteristics of the lowest accuracy and precision. With regard to this biomarker, hypothesising a difference between the exposed and unexposed population of $8 \%$ and a SD of $20 \%$, considering a sample of 150 individuals in each exposure group, and assuming a first-type error of $5 \%$ for bilateral testing, statistical power of $90 \%$ was achieved. As the pilot study on the evaluation of kidney damage is based on a sample halved compared with the main study assessing the cardiovascular risk in the same population, the enrollment ends when for 75 subjects living in each area who have joined the first study, the possibility and the willingness to participate in the second survey is verified.

As the pilot study on the evaluation of kidney damage is based on a sample halved compared with the main study assessing the cardiovascular risk in the same population, the enrollment ends when for 75 subjects living in each area who have joined the first study, the possibility and the willingness to participate in the second survey is verified.

The comparison between the levels of exposure to the selected pollutants and the levels of biological markers will be performed through univariate and multivariate statistical analyses (adjusting for confounding or modification covariates), using parametric and non-parametric statistical methods, chosen after checking the normality of the distribution of the dependent variables considered.

\section{Aims}

The CISAS project embraces multidisciplinary activities with the purpose to deepen knowledge on environmental pollution phenomena and their impact on the ecosystem and human health, in order to plan actions mitigating harmful environmental effects and for preventing risks to human health. 
The three described epidemiological studies in CISAS project, started in January 2019 and currently ongoing, represent the first example of analytical investigations of human biomonitoring based on the evaluation of exposure and preclinical effects in populations residing in NPCSs of southern Italy. The results obtained will allow to develop epidemiological knowledge in the examined areas, and in particular will provide insights in the relationships between priority pollutants of each contaminated site and selected health outcomes, in the view of improving the evidence on and in the molecular mechanisms involved as well. In particular, it will be possible:

- To identify whether subjects exposed to endocrine disrupting chemicals exhibit increased hepatic biomarkers, and alterations of lipid metabolism that may promote the development of liver disease and, with time, possibly HCC.

- To evaluate the association between exposure to heavy metals and increased risk of indicators of thyroid disease and thyroid cancer.

- To assess the effect of exposure to heavy metals on cardiovascular risk and on glomerular and tubular function in cardiovascular primary prevention subjects.

The innovative feature of these studies is represented by the assessment of non-invasive, exposure and earlyeffect biomarkers that would be helpful to implement integrated health-environment surveillance systems in NPCSs and seek novel strategies aimed at decreasing the adverse effects of environmental pollution, including specific remediation programmes of the environmental matrices. Furthermore, all data collected within CISAS project will allow the evaluation of codified cardiovascular risk scores, such as the ACC/AHA atherosclerotic CVD risk score. These scores could be very useful in order to estimate the impact of environmental exposure on future risk of developing CVDs.

Such an approach could be applied in other contaminated sites having similar environment and health characteristics, as it represents a valuable method to monitor exposed communities that could be followed over time for a reliable exposure assessment. Information on early health effects can be particularly useful for planning specific prevention actions for polluted areas.

\section{Patient and public involvement}

The recruitment is based on healthy volunteers adhering inclusion criteria. Patients and the public were not involved in the study design but they are partially involved in the conduction (questionnaires and relationship with the general practitioners) and in the dissemination, as the protocol plans a public presentation of aggregated results to all stakeholders including the patients.

\section{Ethics and dissemination}

The subjects enrolled may participate to the studies after receiving an extensive explanation about project details and research aims, and signing the informed consent.
Each participant is assigned a unique identification code for the chemical tests, instrumental examinations and questionnaires in order to link all the data preserving complete pseudonymisation. All the images acquired are stored on digital support in pseudonymizated form and data are re-evaluated by an operator 'blind' and with quantitative 'operator-independent' systems, in order to reduce interobserver and intraobserver variability. The procedures adopted comply the General Data Protection Regulation (EU) 2016/67 regulating data protection and privacy for all individual citizens of the European Union and the European Economic Area.

In the case of studies based on individual markers of exposure of early-effect specific ethical requirements regulate both the protection of volunteers' privacy and also the dissemination and communication of results. ${ }^{58}$ The content of the questionnaire is developed in accordance with ethical requirements, and is included in the approval procedures of local Ethics Committees. The studies are being conducted in compliance with the principles of Declaration of Helsinki.

Among the requirements, there are the respect of the right to know as the basis of self-determination in the use of research results, the distribution of benefits to different groups and the choice to deliver the results to the research subjects if they want. At enrollment, an information sheet and the informed consent to be signed provide a complete explanation of project details and research aims. Moreover, all the subjects are made aware that they may withdraw from participating at any time. ${ }^{59}$

CISAS has been giving particular importance to the communication of information about the project activities to policy-makers, citizens and stakeholders. ${ }^{60}$ Public conferences have been organised in the previous months and others will be held at the end of the studies to make available the overall results to the local Regional Health Authorities, the authorities of the municipalities involved and the scientific community. ${ }^{60}$ Findings will also be published on international peer-reviewed journals.

On the main topics of the project, educational and training activities in the schools located in the three contaminated areas have been scheduled from before the start to after the end of the studies.

\section{Author affiliations}

${ }^{1}$ National Research Council, Institute of Clinical Physiology, Pisa, Italy

${ }^{2}$ National Research Council, Institute of Clinical Physiology, Reggio Calabria, Italy

${ }^{3}$ Department of Clinical and Experimental Medicine, Section of Pharmacology,

University of Messina, Messina, Italy

${ }^{4}$ Local Health Authority of Messina, Messina, Italy

${ }^{5}$ Fondazione CNR-Regione Toscana G Monasterio, Laboratory Medicine Unit, Pisa, Italy

Twitter Liliana Cori @ilianacori

Acknowledgements The authors sincerely thank Silvia Baldacci, Alessio Coi and Michele Santoro, IFC-CNR, who participated in drafting the protocols and reviewed the manuscript; Alessandro Bisbano, Local Health Authority of Crotone, Fabio Contarino, Lia Contrino and Antonino Colanino Ziino, Local Health Authority of Siracusa, for their support in the acquisition of preliminary data. 
Contributors AG, CV, DB, FB, FF, FM, FS, GT and Ml conceptualised the design and methodology of epidemiological studies; FG drafted the manuscript; FG and $\mathrm{FB}$ critically reviewed the manuscript and gave final approval of the version to be published. CV, DB, EB, FB, FG, FM, FS, GQ, GT, LC and MI contributed in drafting the manuscript. All the authors read and approved the final version of the manuscript.

Funding This research was funded by the Italian Ministry of Education, University and Research, MIUR-Deliberation CIPE n. 105/2015, 23 December 2015, grant number B62F15001070005

Map disclaimer The depiction of boundaries on this map does not imply the expression of any opinion whatsoever on the part of BMJ (or any member of its group) concerning the legal status of any country, territory, jurisdiction or area or of its authorities. This map is provided without any warranty of any kind, either express or implied.

Competing interests None declared.

Patient and public involvement Patients and/or the public were involved in the design, or conduct, or reporting or dissemination plans of this research. Refer to the 'Methods' section for further details.

Patient consent for publication Not required.

Ethics approval The protocol studies have been approved by the Ethics Committees responsible for the three involved NPCSs: the Ethics Committee 'Catania 2' for the NPCS of Priolo (21 July 2017, n. 500/2017/CECT2), the Ethics Committee of the University Hospitals of Messina for the NPCS of MilazzoValle del Mela (19 February 2018, n.2/2018) and the Ethics Committee of the Region of Calabria for the NPCS of Crotone (20 July 2017, n. 174). Results will be disseminated among policy-makers, citizens, stakeholders and scientific community through the organisation of conferences and events, and the publication on international peer/reviewed journals.

Provenance and peer review Not commissioned; externally peer reviewed.

Open access This is an open access article distributed in accordance with the Creative Commons Attribution Non Commercial (CC BY-NC 4.0) license, which permits others to distribute, remix, adapt, build upon this work non-commercially, and license their derivative works on different terms, provided the original work is properly cited, appropriate credit is given, any changes made indicated, and the use is non-commercial. See: http://creativecommons.org/licenses/by-nc/4.0/.

ORCID iD

Francesca Gorini http://orcid.org/0000-0002-4619-6227

\section{REFERENCES}

1 Press-Ustün A, Wolf J, Corvalán C. Preventing disease through healthy environments. Geneva: World Health Organization, 2016: 1-176. https://apps.who.int/iris/bitstream/handle/10665/ 204585/9789241565196_eng.pdf?sequence=1\&isAllowed=y

2 World Health Organization. Regional office for Europe. sixth Ministerial conference on environment and health. Available: http:// www.euro.who.int/en/media-centre/events/events/2017/06/sixthministerial-conference-on-environment-and-health/read-more [Accessed 8 July 2019].

3 Perez AP, Eugenio NR. Status of local soil contamination in Europe: Revision of the indicator "Progress in the management Contaminated Sites in Europe, EUR 29124 EN. Luxembourg: Publications Office of the European Union, 2018: 1-293.

4 Zona A, lavarone I, Buzzoni C, et al. [SENTIERI: Epidemiological Study of Residents in National Priority Contaminated Sites. Fifth Report]. Epidemiol Prev 2019;43:1-208.

5 Agency EE. Costs of air pollution from European industrial facilities 2008-2012 - an updated assessment. EEA technical report no 20/2014. Available: https://www.eea.europa.eu/publications/costsof-air-pollution-2008-2012 [Accessed 8 July 2019]

6 National Institute for the Environmental Protection and Research. National priority contaminated sites. Available: https://annuario. isprambiente.it/ada/basic/6798 [Accessed 8 July 2019].

7 Pirastu R, Ancona C, lavarone I, et al. SENTIERI project. mortality study of residents in Italian polluted sites: evaluation of the epidemiological evidence. Epidemiol Prev 2010;34:1-2.

8 Martin-Olmedo P, Ranzi A, Santoro M, et al. Methods and data needs to assess health impacts of chemicals in industrial contaminated sites. Epidemiol Prev 2019;43:223-37.

9 Ruggieri S, Drago G, Colombo P, et al. Three contaminated sites in southern Italy. the neonatal environment and health outcomes cohort: protocol for a longitudinal birth cohort study. BMJ Open 2019:9:e029471.

10 Fazzo L, Carere M, Tisano F, et al. Cancer incidence in Priolo, Sicily: a spatial approach for estimation of industrial air pollution impact. Geospat Health 2016;11:320.

11 Pirastu R, Comba R, Conti S, et al. SENTIERI - Epidemiological Study of Residents in National Priority Contaminated Sites: mortality, cancer incidence and hospital discharges. Epidemiol Prev 2014;38:1-170.

12 Park J-W, Chen M, Colombo M, et al. Global patterns of hepatocellular carcinoma management from diagnosis to death: the bridge study. Liver Int 2015;35:2155-66.

13 Rapisarda V, Loreto C, Malaguarnera M, et al. Hepatocellular carcinoma and the risk of occupational exposure. World $\mathrm{J}$ Hepatol 2016;8:573-90.

14 Rahman R, Hammoud GM, Almashhrawi AA, et al. Primary hepatocellular carcinoma and metabolic syndrome: an update. World $J$ Gastrointest Oncol 2013;5:186-94.

15 Huang C, Freter C. Lipid metabolism, apoptosis and cancer therapy. Int J Mol Sci 2015;16:924-49.

16 Pellegriti G, Frasca F, Regalbuto C, et al. Worldwide increasing incidence of thyroid cancer: update on epidemiology and risk factors. J Cancer Epidemiol 2013;2013:1-10.

17 Zaccarelli-Marino MA, Alessi R, Balderi TZ, et al. Association between the occurrence of primary hypothyroidism and the exposure of the population near to industrial pollutants in São Paulo state, Brazil. Int J Environ Res Public Health 2019;16:3464.

18 Gorini F, lervasi G, Coi A, et al. The role of polybrominated diphenyl ethers in thyroid carcinogenesis: is it a weak hypothesis or a hidden reality? from facts to new perspectives. Int J Environ Res Public Health 2018:15:1834.

19 Nettore IC, Colao A, Macchia PE. Nutritional and environmental factors in thyroid carcinogenesis. Int J Environ Res Public Health 2018;15. doi:10.3390/ijerph15081735. [Epub ahead of print: 13 Aug 2018].

20 Interdonato M, Bitto A, Pizzino G, et al. Levels of heavy metals in adolescents living in the industrialised area of Milazzo-Valle del Mela (Northern Sicily). J Environ Public Health 2014;2014:1-9.

21 Nie X, Chen Y, Chen Y, et al. Lead and cadmium exposure, higher thyroid antibodies and thyroid dysfunction in Chinese women. Environ Pollut 2017;230:320-8.

22 Stojsavljević A, Rovčanin B, Krstić Đurđa, et al. Cadmium as main endocrine disruptor in papillary thyroid carcinoma and the significance of $\mathrm{Cd} / \mathrm{Se}$ ratio for thyroid tissue pathophysiology. J Trace Elem Med Biol 2019;55:190-5.

23 Jancic SA, Stosic BZ. Cadmium effects on the thyroid gland. Vitam Horm 2014;94:391-425.

24 Comba P, Pitimada M. Studio epidemiologico dei siti contaminati DELLA Calabria: obiettivi, metodologia, fattibilit. Roma: Istituto Superiore di Sanit, 2016

25 Jomova K, Jenisova Z, Feszterova M, et al. Arsenic: toxicity, oxidative stress and human disease. J App/ Toxicol 2011;31:n/a-107.

26 Jomova K, Valko M. Advances in metal-induced oxidative stress and human disease. Toxicology 2011;283:65-87.

27 Chowdhury R, Ramond A, O'Keeffe LM, et al. Environmental toxic metal contaminants and risk of cardiovascular disease: systematic review and meta-analysis. BMJ 2018;362:k3310.

28 Lentini P, Zanoli L, Granata A, et al. Kidney and heavy metals - The role of environmental exposure (Review). Mol Med Rep 2017:15:3413-9.

29 Orr SE, Bridges CC. Chronic kidney disease and exposure to nephrotoxic metals. Int J Mol Sci 2017;18:pii: E1039.

30 Navas-Acien A, Tellez-Plaza M, Guallar E, et al. Blood cadmium and lead and chronic kidney disease in US adults: a joint analysis. Am J Epidemiol 2009;170:1156-64.

31 Kim NH, Hyun YY, Lee K-B, et al. Environmental heavy metal exposure and chronic kidney disease in the general population. $J$ Korean Med Sci 2015;30:272-7.

32 Sayiner M, Golabi P, Younossi ZM. Disease burden of hepatocellular carcinoma: a global perspective. Dig Dis Sci 2019;64:910-7.

33 Ferlay J, Soerjomataram I, Dikshit R, et al. Cancer incidence and mortality worldwide: sources, methods and major patterns in GLOBOCAN 2012. Int J Cancer 2015;136:E359-86.

34 Interdonato M, Pizzino G, Bitto A, et al. Cadmium delays puberty onset and testis growth in adolescents. Clin Endocrinol 2015;83:357-62.

35 Arnett DK, Blumenthal RS, Albert MA, et al. 2019 ACC/AHA guideline on the primary prevention of cardiovascular disease: a report of the American College of Cardiology/American heart association Task force on clinical practice guidelines. Circulation 2019;140:e596-646. 
36 GBD 2017 Disease and Injury Incidence and Prevalence Collaborators. Global, regional, and national incidence, prevalence, and years lived with disability for 354 diseases and injuries for 195 countries and territories, 1990-2017: a systematic analysis for the global burden of disease study 2017. Lancet 2018;392:1789-858.

37 Jagannathan R, Patel SA, Ali MK, et al. Global updates on cardiovascular disease mortality trends and Attribution of traditional risk factors. Curr Diab Rep 2019;19:44.

38 Otgonsuren M, Estep MJ, Hossain N, et al. Single non-invasive model to diagnose non-alcoholic fatty liver disease (NAFLD) and non-alcoholic steatohepatitis (NASH). J Gastroenterol Hepatol 2014;29:2006-13.

39 Bedogni G, Bellentani S, Miglioli L, et al. The fatty liver index: a simple and accurate predictor of hepatic steatosis in the general population. BMC Gastroenterol 2006:6:33.

40 Castera L, Friedrich-Rust M, Loomba R. Noninvasive assessment of liver disease in patients with nonalcoholic fatty liver disease. Gastroenterology 2019;156:1264-81.

41 European Association for the Study of the Liver (EASL), European Association for the Study of Diabetes (EASD), European Association for the Study of Obesity (EASO). EASL-EASD-EASO clinical practice guidelines for the management of non-alcoholic fatty liver disease. $J$ Hepatol 2016;64:1388-402.

42 Sterling RK, Lissen E, Clumeck N, et al. Development of a simple noninvasive index to predict significant fibrosis in patients with HIV/ HCV coinfection. Hepatology 2006;43:1317-25.

43 Angulo P, Hui JM, Marchesini G, et al. The NAFLD fibrosis score: a noninvasive system that identifies liver fibrosis in patients with NAFLD. Hepatology 2007;45:846-54.

44 Bitto A, Polito F, Atteritano M, et al. Genistein aglycone does not affect thyroid function: results from a three-year, randomized, double-blind, placebo-controlled trial. J Clin Endocrinol Metab 2010;95:3067-72.

45 Houston MC. Role of mercury toxicity in hypertension, cardiovascular disease, and stroke. J Clin Hypertens 2011;13:621-7.

46 Alissa EM, Ferns GA. Heavy metal poisoning and cardiovascular disease. J Toxicol 2011;2011:1-21.

47 Sangartit W, Kukongviriyapan U, Donpunha W, et al. Tetrahydrocurcumin protects against cadmium-induced hypertension, raised arterial stiffness and vascular remodeling in mice. PLoS One 2014;9:e114908.
48 Kaya Y, Ari E, Demir H, et al. Serum cadmium levels are independently associated with endothelial function in hemodialysis patients. Int Urol Nephrol 2012;44:1487-92.

49 Scimeca M, Feola M, Romano L, et al. Heavy metals accumulation affects bone microarchitecture in osteoporotic patients. Environ Toxicol 2017;32:1333-42.

50 Vassalle C, Mazzone A. Bone loss and vascular calcification: a bidirectional interplay? Vascul Pharmacol 2016;86:77-86.

51 Vassalle C, lervasi G. Cathepsin K--a classical bone biomarker in cardiovascular disease: the heart is not alone anymore. Atherosclerosis 2013;228:36-7.

52 Botev R, Mallié J-P, Couchoud C, et al. Estimating glomerular filtration rate: Cockcroft-Gault and modification of diet in renal disease formulas compared to renal inulin clearance. Clin J Am Soc Nephrol 2009;4:899-906.

53 Stevens LA, Coresh J, Schmid CH, et al. Estimating GFR using serum cystatin $\mathrm{C}$ alone and in combination with serum creatinine: a pooled analysis of 3,418 individuals with CKD. Am J Kidney Dis 2008;51:395-406.

54 Haugen BR, Alexander EK, Bible KC, et al. 2015 American thyroid association management guidelines for adult patients with thyroid nodules and differentiated thyroid cancer: the American thyroid association guidelines Task force on thyroid nodules and differentiated thyroid cancer. Thyroid 2016;26:1-133.

55 Bianchini E, Giannarelli C, Bruno RM, et al. Functional and structural alterations of large arteries: methodological issues. Curr Pharm Des 2013;19:2390-400.

56 Thijssen DHJ, Bruno RM, van Mil ACCM, et al. Expert consensus and evidence-based recommendations for the assessment of flowmediated dilation in humans. Eur Heart J 2019;40:2534-47.

57 Walsh JP. Managing thyroid disease in general practice. Med J Aust 2016;205:179-84.

58 Van Damme K, Casteleyn L. Biomonitoring and health surveillance: ethical challenges. G Ital Med Lav Ergon 2010;32:79-82.

59 Kramer S, Soskolne CL, Mustapha BA, et al. Revised ethics guidelines for environmental epidemiologists. Environ Health Perspect 2012;120:a299-301.

60 Cori L, Bianchi F, Sprovieri M, et al. Communication and community involvement to support risk governance. Int J Environ Res Public Health 2019;16:4356. 